\title{
Bragg onion resonators with omnidirectional reflector cladding
}

Yong Xu, Wei Liang, James G. Fleming, Yanyi Huang, Amnon Yariv, et al.

Yong $\mathrm{Xu}$, Wei Liang, James G. Fleming, Yanyi Huang, Amnon Yariv, Shawn-Yu Lin, "Bragg onion resonators with omnidirectional reflector cladding," Proc. SPIE 5360, Photonic Crystal Materials and Devices II, (9 July 2004); doi: 10.1117/12.553910

SPIE Event: Integrated Optoelectronic Devices 2004, 2004, San Jose, CA, United States 


\title{
Bragg onion resonators with omnidirectional reflector cladding
}

\author{
Yong Xu, ${ }^{a}$ Wei Liang, ${ }^{a}$ James G. Fleming, ${ }^{b}$ Yanyi Huang, ${ }^{a}$ Amnon Yariv,${ }^{a}$ and Shawn Y. $\operatorname{Lin}^{b}$ \\ ${ }^{a}$ Department of Applied Physics, MS 128-95, \\ California Institute of Technology, Pasadena, CA 91125, USA; \\ ${ }^{b}$ Sandia National Laboratories, Albuquerque, NM 87185-1080, USA.
}

\begin{abstract}
We propose to use onion-like resonators to approximate spherically symmetric Bragg resonators. Such Bragg onion resonators have been realized in silicon based material systems. We develop an analytical theory that calculates the resonant frequencies and the quality factors of the onion cavity modes. We demonstrate that it is possible to achieve $\mathrm{Q}$ factors exceeding $5 \times 10^{6}$ in a cavity of a few microns in dimension. The onion resonators allow full control over the spontaneous emission process, which may lead to the thresholdless lasers. The onion resonators may also find many other applications in cavity quantum electrodynamics.
\end{abstract}

Keywords: Photonic Crystals, Bragg resonators, Optical Microcavities, Cavity Quantum Electrodynamics.

\section{INTRODUCTION}

It has been known for some time that spontaneous emission can be significantly modified by the presence of an optical microcavity. ${ }^{1}$ With advancements in microfabrication techniques, there has been considerable interest in creating an optical cavity with a small modal volume and a large Q factor. In such a cavity, it is possible to funnel a significant portion of the spontaneous emission into a desired cavity mode, which can be characterized by the spontaneous emission factor $\beta$, defined as the fraction of spontaneous emission radiated into a given optical mode. In the extreme case of $\beta$ approaching unity, we can achieve "thresholdless" lasing in such a cavity. ${ }^{2}$ An optical cavity with a large spontaneous emission factor $\beta$ is also critical for realizing single photon devices. ${ }^{3}$ Furthermore, an optical cavity that combines a small modal volume with a large Q factor, which can significantly enhance the coupling between the atom and the cavity mode, is also of great interest to the community of cavity quantum electrodynamics (QED). ${ }^{4}$

Some potential candidates in the literature that can be useful for spontaneous emission engineering are silica microspheres, ${ }^{5}$ semiconductor microdisks, ${ }^{6}$ semiconductor pillars, ${ }^{3}$ and defect cavities in photonic crystals. ${ }^{7}$ The whispering-gallery modes in silica microspheres can achieve extremely high quality factors of up to $10^{9}$. However, the radius of the silica microsphere is limited to be above $10 \mu \mathrm{m}$, and can not be decreased much further without suffering a rapidly decreasing cavity $\mathrm{Q}$ factor. In the case of semiconductor micropillars or photonic crystal defect cavities, the modal volume can become very small (of the order of $\left.(\lambda / n)^{3}\right)$. Yet fabrication imprecision would limit the $\mathrm{Q}$ factors to a much lower value of a few thousands. ${ }^{3}$ Recently, a few theoretical discussions of Bragg resonators with perfect spherical symmetry have been presented. ${ }^{8-10}$ Such resonators, however, are difficult to realize. In this paper, we propose to approximate such spherically symmetric Bragg resonators with an onion-like geometry (as shown in Fig. 1). In following sections, we shall demonstrate that the Bragg onion resonators can combine a high $\mathrm{Q}$ factor with a cavity spatial dimension of a few microns, and as a result, allows dramatic modification of the spontaneous emission process in such cavities.

As shown in Fig. 1, the Bragg onion resonator consists of a spherical low index core (possibly air) surrounded by alternating concentric dielectric layers with different refractive indices. We notice that the Bragg onion resonator can be regarded as a three-dimensional analog of a Bragg fiber, which was first proposed in $1978,{ }^{11}$ and has recently attracted much attention. ${ }^{12-14}$

Yong Xu's email address is yong@its.caltech.edu. 


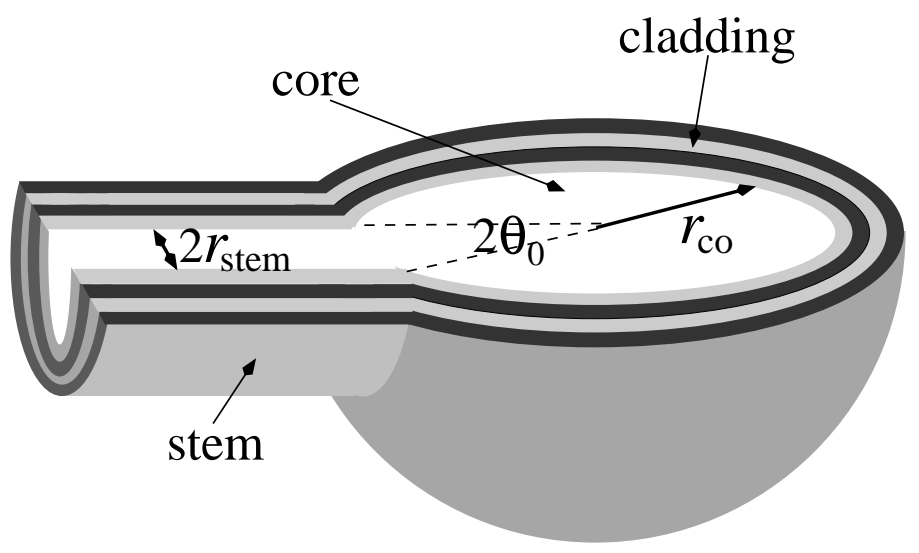

Figure 1. Schematics of a Bragg onion resonator.

The Bragg onion resonators have been realized in silicon based materials. A scanning electron microscope (SEM) image of a Bragg onion resonator is shown in Fig. 2. The fabrication process of the Bragg onion resonator begins with the introduction of a cylindrical opening in a silicon wafer, which also defines the onion stem in Fig. 1. Subsequently, isotropic etching is used to create a hollow spherical cavity at the end of the stem section. Finally, with chemical vapor deposition, the Bragg mirror stacks can be constructed by the deposition of Si from silane, $\mathrm{Si}_{3} \mathrm{~N}_{4}$ from dichlorosilane and ammonia, and $\mathrm{SiO}_{2}$ from the thermal oxidation of silicon. A particular appealing advantage of the onion design is that we can introduce a wide range of light emitters into the hollow cavity. Additionally, by choosing a Bragg cladding composed of $\mathrm{Si} / \mathrm{SiO}_{2}$ pairs, which have a large index contrast, we can construct an omnidirectional reflector cladding, which is important for engineering spontaneous emission, as we shall see later.

\section{TRANSFER MATRIX FORMALISM}

In the following analysis, we assume a time dependence of $e^{-i \omega t}$ and allow the frequency $\omega$ to be a complex number, whose imaginary part accounts for the modal loss. In spherical coordinates, we can express the general solution to the Maxwell equations in the $n$th concentric dielectric layer as a superposition of various multipole components, each characterized by the angular quantum number $l$ and $m::^{15}$

$$
\begin{gathered}
\vec{H}=\sum_{l} \sum_{m=-l}^{l}\left[f_{l}\left(k_{n} r\right) \vec{X}_{l m}-\frac{i}{k_{n}} \vec{\nabla} \times\left(g_{l}\left(k_{n} r\right) \vec{X}_{l m}\right)\right], \\
\vec{E}=Z_{n} \sum_{l} \sum_{m=-l}^{l}\left[\frac{i}{k_{n}} \vec{\nabla} \times\left(f_{l}\left(k_{n} r\right) \vec{X}_{l m}\right)+g_{l}\left(k_{n} r\right) \vec{X}_{l m}\right],
\end{gathered}
$$

where $\epsilon_{n}$ is the relative dielectric constant of the $n$th layer, $Z_{n}=\sqrt{\mu_{0} / \epsilon_{0} \epsilon_{n}}$ is the material impedance, and $k_{n}=\sqrt{\epsilon_{n}} \omega / c$. The angular dependence of the solution is described by $\vec{X}_{l m}$ :

$$
\vec{X}_{l m}=\frac{1}{i \sqrt{l(l+1)}}\left[-\hat{e}_{\theta} \frac{1}{\sin \theta} \frac{\partial}{\partial \phi}+\hat{e}_{\phi} \frac{\partial}{\partial \theta}\right] Y_{l m}(\theta, \phi) .
$$

where $Y_{l m}(\theta, \phi)$ is the $(1, \mathrm{~m})$ spherical harmonic. Since $\vec{X}_{l m}$ has no $\hat{e}_{r}$ component, the first term on the right hand side of Eq. (1) and Eq. (2) represents the transverse magnetic (TM) component, whereas the second term 

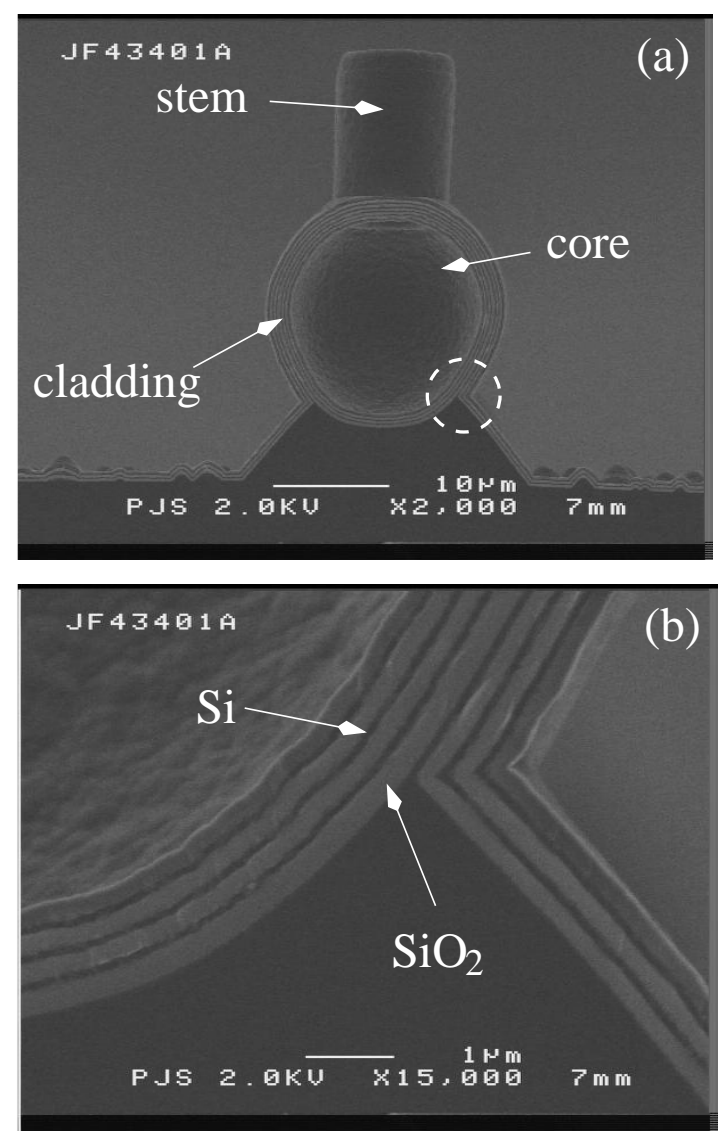

Figure 2. (a) A SEM image of an air core Bragg onion resonator with $\mathrm{Si} / \mathrm{SiO}_{2}$ cladding pairs. (b) A SEM image of the onion resonator cladding, which corresponds to the region within the dashed circle in (a).

represents the transverse electric (TE) component. In Eqs. (1) and (2), the radial dependence of the solution is given by ${ }^{15}$

$$
\begin{aligned}
& f_{l}\left(k_{n} r\right)=A_{n} h_{l}^{(1)}\left(k_{n} r\right)+B_{n} h_{l}^{(2)}\left(k_{n} r\right), \\
& g_{l}\left(k_{n} r\right)=C_{n} h_{l}^{(1)}\left(k_{n} r\right)+D_{n} h_{l}^{(2)}\left(k_{n} r\right),
\end{aligned}
$$

where $h_{l}^{(1)}$ and $h_{l}^{(2)}$ are, respectively, the first and second kind of spherical Hankel function. The coefficients $A_{n}$, $B_{n}, C_{n}$, and $D_{n}$ are constant within the $n$th dielectric layer. The coefficients in the $n+1$ th layer can be related to those in the $n$th layer via the continuity of $E_{\theta}, E_{\phi}, H_{\theta}$, and $H_{\phi}$ at $r=r_{n}$, the interface between the adjacent layers. Combining Eqs. (1) to (5) and applying the orthogonality of the spherical harmonics, we find that for a given multipole component, the following matrix relation holds

$$
\begin{array}{r}
{\left[\begin{array}{cc}
h_{l}^{(1)}\left(k_{n} r_{n}\right) & h_{l}^{(2)}\left(k_{n} r_{n}\right) \\
\left.\frac{Z_{n}}{k_{n}} \frac{\partial\left[r h_{l}^{(1)}\left(k_{n} r\right)\right]}{\partial r}\right|_{r_{n}} & \left.\frac{Z_{n}}{k_{n}} \frac{\partial\left[r h_{l}^{(2)}\left(k_{n} r\right)\right]}{\partial r}\right|_{r_{n}}
\end{array}\right]\left[\begin{array}{c}
A_{n} \\
B_{n}
\end{array}\right]=} \\
{\left[\begin{array}{cc}
h_{l}^{(1)}\left(k_{n+1} r_{n}\right) & h_{l}^{(2)}\left(k_{n+1} r_{n}\right) \\
\left.\frac{Z_{n+1}}{k_{n+1}} \frac{\partial\left[r h_{l}^{(1)}\left(k_{n+1} r\right)\right]}{\partial r}\right|_{r_{n}} & \left.\frac{Z_{n+1}}{k_{n+1}} \frac{\partial\left[r h_{l}^{(2)}\left(k_{n+1} r\right)\right]}{\partial r}\right|_{r_{n}}
\end{array}\right]\left[\begin{array}{c}
A_{n+1} \\
B_{n+1}
\end{array}\right],}
\end{array}
$$




$$
\begin{array}{r}
{\left[\begin{array}{cc}
Z_{n} h_{l}^{(1)}\left(k_{n} r_{n}\right) & Z_{n} h_{l}^{(2)}\left(k_{n} r_{n}\right) \\
\left.\frac{1}{k_{n}} \frac{\partial\left[r h_{l}^{(1)}\left(k_{n} r\right)\right]}{\partial r}\right|_{r_{n}} & \left.\frac{1}{k_{n}} \frac{\partial\left[r h_{l}^{(2)}\left(k_{n} r\right)\right]}{\partial r}\right|_{r_{n}}
\end{array}\right]\left[\begin{array}{c}
C_{n} \\
D_{n}
\end{array}\right]=} \\
{\left[\begin{array}{cc}
Z_{n+1} h_{l}^{(1)}\left(k_{n+1} r_{n}\right) & Z_{n+1} h_{l}^{(2)}\left(k_{n+1} r_{n}\right) \\
\left.\frac{1}{k_{n+1}} \frac{\partial\left[r h_{l}^{(1)}\left(k_{n+1} r\right)\right]}{\partial r}\right|_{r_{n}} & \left.\frac{1}{k_{n+1}} \frac{\partial\left[r h_{l}^{(2)}\left(k_{n+1} r\right)\right]}{\partial r}\right|_{r_{n}}
\end{array}\right]\left[\begin{array}{c}
C_{n+1} \\
D_{n+1}
\end{array}\right],}
\end{array}
$$

From Eq. (6) and Eq. (7) it follows that: (i) multipole components with different $l$ and $m$ are independent of each other, a direct result of the spherical symmetry of the onion structure, and (ii) TE components and TM components decouple from each other. Consequently, we can denote various optical modes in the onion structure as $\mathrm{TE}_{l m}$ or $\mathrm{TM}_{l m}$, according to their angular quantum number $l$ and $m$, and the polarization state (i.e. $\mathrm{TE}$ or TM).

For a Bragg onion resonator with a finite number of cladding layers, we can apply Eqs. (6) and (7) iteratively to relate the coefficients within the onion core $\left(A_{c o}, B_{c o}, C_{c o}\right.$, and $\left.D_{c o}\right)$ to those outside of the onion $\left(A_{\text {out }}, B_{\text {out }}\right.$, $C_{\text {out }}$, and $\left.D_{\text {out }}\right)$. For a TM mode, this leads to

$$
\left[\begin{array}{l}
A_{\text {out }} \\
B_{\text {out }}
\end{array}\right]=\left[\begin{array}{ll}
T_{11} & T_{12} \\
T_{21} & T_{22}
\end{array}\right]\left[\begin{array}{l}
A_{c o} \\
B_{c o}
\end{array}\right]
$$

The onion cavity modes should satisfy the following boundary conditions: (i) the core electromagnetic must be finite, therefore $A_{c o}=B_{c o}$ and $C_{c o}=D_{c o}$, (ii) only the outgoing wave exists outside of the onion, which gives $B_{\text {out }}=0$ and $D_{\text {out }}=0$. Substituting these two condition into Eq. (8), we find that

$$
T_{21}+T_{22}=0,
$$

must be satisfied for the onion cavity mode. Thus the search for the resonant mode of the onion cavity is mathematically equivalent to finding the complex $\omega$ that satisfies Eq. (9). The real part of the solution is the modal frequency, and the imaginary part gives the cavity Q factor via $Q=\operatorname{Re}(\omega) /[2 \operatorname{Im}(\omega)]$.

One immediate result of this theoretical framework is that for any given angular momentum $l$, there are $2 l+1$ degenerate high $\mathrm{Q}$ modes in the onion cavity, with not only identical resonant frequency but also the same quality factor. This follows directly from the full $\mathrm{SO}(3)$ rotational symmetry of the onion structure. Another interesting result is that the lowest order of "angular momentum" allowed is $l=1$ rather than $l=0$, since $Y_{00}(\theta, \phi)$ is a constant, which leads to a null vector $\vec{X}_{00}$ (see Eq. (3)). This comes from the fact that the lowest order of electromagnetic radiation is dipole radiation.

\section{RESULTS AND DISCUSSIONS}

We now apply this formalism to investigate the modal spectrum of a specific onion structure. Our example has an air core (radius $r_{c o}=3.47 \mu \mathrm{m}$ ) surrounded by 8 Bragg cladding pairs. Each cladding pair is composed of a polysilicon layer with refractive index $n_{S i}=3.5$ and thickness $L_{S i}=0.111 \mu m$, followed by a layer of $\mathrm{SiO}_{2}$ with refractive index $n_{\mathrm{SiO}_{2}}=1.5$ and thickness $L_{\mathrm{SiO}_{2}}=0.258 \mu \mathrm{m}$. In this design, the cladding layers form quarter wave stack at $\lambda \approx 1.55 \mu \mathrm{m}$. In Fig. (3a) and (3b), we show the quality factor of the onion modes with $l \leq 7$, in the wavelength range of $1.35 \mu m \leq \lambda \leq 1.70 \mu \mathrm{m}$. Notice that with only 8 pairs of Bragg cladding layers, we can achieve cavity $\mathrm{Q}$ values exceeding $5 \times 10^{6}$. In Fig. (3c), we plot the $\mathrm{Q}$ factors of $\mathrm{TM}_{20}$ modes as a function of Bragg cladding pair number $N_{\text {clad }}$ and find a excellent exponential dependence of $Q \propto D^{N_{\text {clad }}}$. Fitting the numerical results, we find a constant of $D_{T M 20}=5.41$ for the TM20 mode. This factor is very close to $\left(n_{\mathrm{Si}} / n_{\mathrm{SiO}_{2}}\right)^{2}$, which equals to 5.44. This similarity can be attributed to the fact that the "leakage" through the quarter wave stack of the onion cladding is reduced by a factor of $\left(n_{\mathrm{Si}_{i}} / n_{\mathrm{SiO}_{2}}\right)^{2}$ with each additional $\mathrm{Si} / \mathrm{SiO}_{2}$ pair, ${ }^{16}$ which improves the quality factor by the same amount.

In Fig. 4, we show the radial dependence of the $\mathrm{TE}_{50}$ and $\mathrm{TE}_{70}$ modes. For both modes, we observe that the electric field is zero at the air-Si interface, similar to the case of a hollow spherical metallic cavity. This is not a coincidence, since the onion cladding forms an omnidirectional reflector around $\lambda=1.55 \mu \mathrm{m}$ and behaves like a perfect metal. ${ }^{17}$ The bandgap of the onion cladding layer is shown in Fig. 5 , where the 


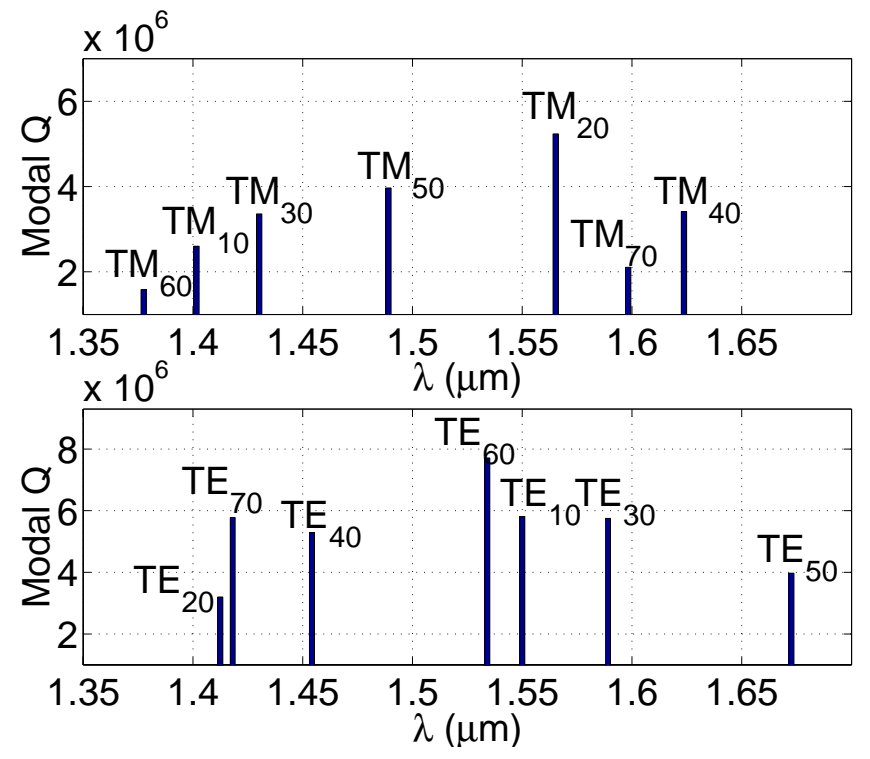

(a)

(b)

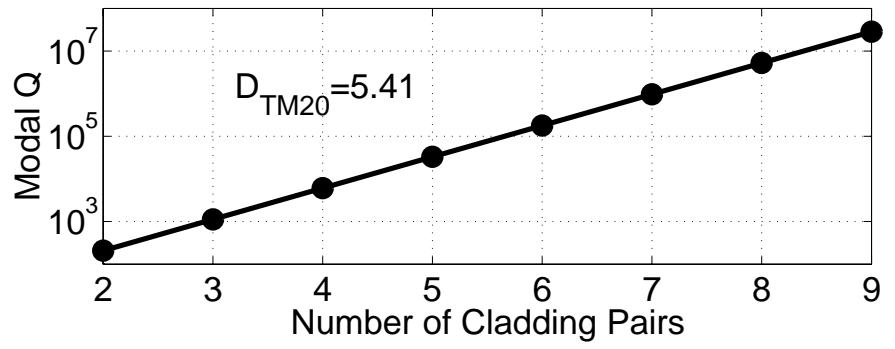

(c)

Figure 3. (a), (b) Wavelength and quality factor of the onion resonator modes. (c) Quality factor of the $\mathrm{TM}_{20} \mathrm{modes}$ as a function of the Bragg cladding pair number. The circles represents the analytical results, whereas the solid lines are given by the numerical fit of $Q \propto D^{N_{c l a d}}$.

existence of an omnidirectional bandgap is clearly shown. The frequency range of the omnidirectional gap is $1.23 \mu m<\lambda<1.62 \mu m$.

There is, however, an important difference between the metallic cavity and the omnidirectional cavity: metals have finite conductivity. For a hollow spherical metallic cavity with a finite skin depth $\delta$ and core radius $r_{c o}$, the cavity $\mathrm{Q}$ factor is roughly given by $r_{c o} / \delta{ }^{15}$ If we use $r_{c o}=3.47 \mu \mathrm{m}$ and take $\delta$ to be that of gold (about a few nanometers in the infrared range), we find that the $\mathrm{Q}$ factor of a metallic cavity is limited to about 1000, much less than what is possible in a dielectric onion cavity. From another point of view, if we take the onion cavity Q to be $3 \times 10^{6}$ and back-calculate the effective skin depth of the Bragg cladding, we find that it behaves as a metallic material with the skin depth of $0.01 \AA$, far less than the radius of an atom! A further consequence of the perfect metal analogy is that the Bragg cladding completely isolates the onion core from the outside free space, and can greatly enhance or inhibit the spontaneous emission, depending on whether the light emitter is on or off the cavity resonance.

As shown in Fig. 2, the Bragg onion resonator has one stem. The presence of the stem breaks the spherical symmetry and lifts the degeneracy of the cavity modes. To characterize the impact of the stems on the cavity loss, we introduce the quantity $Q_{\text {stem }}$, defined as $\omega E_{\text {cavity }} / P_{\text {stem }}$, where $E_{\text {cavity }}$ is the electromagnetic energy stored in the onion core and $P_{\text {stem }}$ is the power leakage through the onion stem. If $Q_{\text {stem }}$ is much larger than the $\mathrm{Q}$ factor of the onion without the stem, we can ignore the presence of the stem in Fig. 1 and Fig. 2, and treat it as a perfectly spherical onion. Whereas if the opposite is true, then the cavity loss is dominated by the photon "leakage" through the onion stem. 


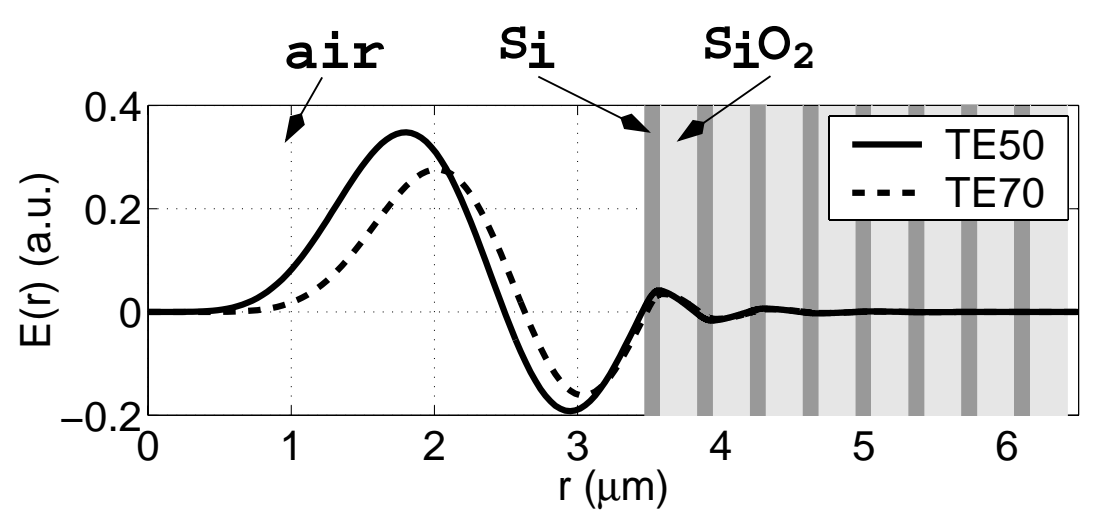

Figure 4. The radial dependence of the $\mathrm{TE}_{10}$ and $\mathrm{TE}_{50}$ field.

From the previous analysis, we can picture the optical fields within the onion core as composed of an incoming and an outgoing spherical wave with equal amplitude but in opposite directions, whose angular radiation pattern is given by $\left|\vec{X}_{l m}(\theta, \phi)\right|^{2}{ }^{15}$ For onions with core size comparable to the photon wavelength, we can estimate $Q_{\text {stem }}$ as $1 /\left[\left|\vec{X}_{l m}\left(\theta_{0}, \phi\right)\right|^{2} \Delta \Omega_{\text {stem }}\right]$, where $\theta_{0}$ is defined in Fig. 1 and $\Delta \Omega_{\text {stem }}$ is the solid angle spanned by the stems. From this expression, we find that among the "dipole" modes $(l=1)$, the one with $m=0$ has the lowest stem loss, with $Q_{\text {stem }}=4 /\left(3 \theta_{0}^{4}\right)$ (assuming $\left.\theta_{0} \ll \pi / 2\right)$. Whereas for $l \geq 2$, those with $m= \pm l$ have the highest $Q_{\text {stem }}$, which is given by

$$
Q_{\text {stem }}=\frac{(l+1)\left(2^{l} l !\right)^{2}}{(2 l+1) ! l} \frac{1}{\theta_{0}^{2 l}} .
$$

Assuming a stem radius of $0.5 \mu \mathrm{m}$, a core radius of $3.47 \mu \mathrm{m}$, we find that for the resonant modes with $l>4$ and $m= \pm l$, the $Q_{\text {stem }}$ exceeds $10^{7}$, which means that the presence of the onion stem has little impact on the cavity Q factors.

A particular appealing advantage of the onion structure shown in Fig. 1 and Fig. 2 is that we can introduce a wide range of light emitters, such as gas-phase neutral atoms and organic molecules, into the onion core. If the light emission wavelength coincides with the cavity resonance, the light emitters will experience enhanced spontaneous emission. Under some general assumptions, it can be shown that the spontaneous emission rate into the cavity mode $\gamma_{c a v}^{s p}$ can be approximated by ${ }^{18}$

$$
\gamma_{\text {cav }}^{s p}=\left[\frac{1}{4 \pi^{2}} \frac{\lambda^{3}}{V_{\text {cav }}} \frac{1}{\frac{1}{Q}+\frac{\gamma_{a}}{\omega}}\right] \gamma_{\text {free }}^{s p},
$$

where $\lambda$ is the optical wavelength, $\omega$ is the optical frequency, $V_{\text {cav }}$ is the cavity volume, $\gamma_{a}$ is the dephasing rate of the light emitter, and $\gamma_{\text {free }}^{s p}$ is the free space spontaneous emission rate. In the regime of "field dominated decay" ( $\gamma_{a} \ll \omega / Q$, such as the case of atomic transition), the onion structure analyzed in Fig. 3 can lead to a 3000-fold cavity enhancement of the spontaneous emission rate and, correspondingly, a spontaneous emission factor approaching unity. In contrast, the state of the art in spontaneous emission enhancement is about a factor of 5 in the optical frequency range. ${ }^{3,6}$ If, on the other hand, the light emission wavelength is outside any of the cavity resonances, the only other possible modes are the cladding modes, which are mainly confined in the onion cladding via total internal reflection and have little intensity in the core region. Consequently, a large spontaneous emission inhibition would occur in this off-resonance case. In this aspect, the onion cavity resembles more a defect cavity in a three-dimensional photonic crystal, ${ }^{19}$ which can also significantly enhance or inhibit spontaneous emission. However, the onion structures enjoy considerable advantage in its design simplicity and a fabrication process that is relatively easier and more controllable. 


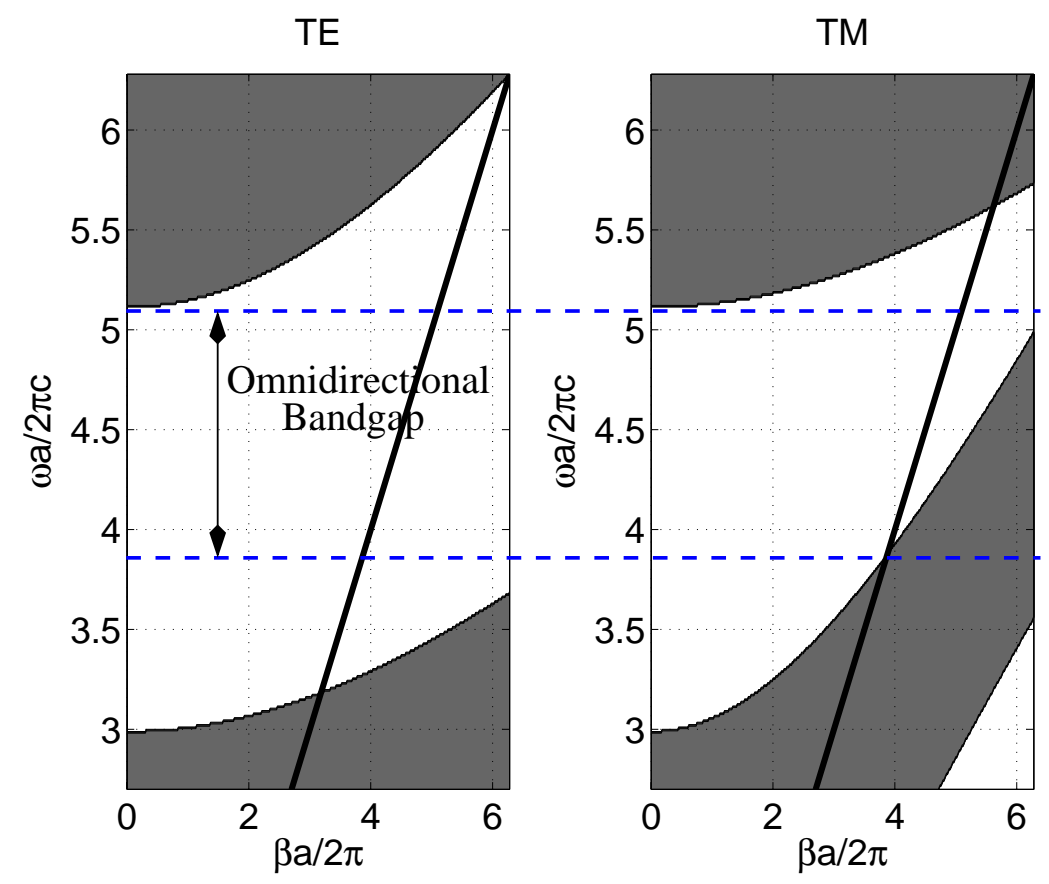

Figure 5. Band diagram of the $\mathrm{Si} / \mathrm{SiO}_{2}$ cladding layers of the Bragg onion resonator. The parameters of the cladding layers correspond to the values given in the text. The shaded region indicates the existence of propagating modes in the Bragg cladding layers. The straight lines are the light line $(\beta=\omega / c)$. The dashed lines indicates the range of omnidirectional bandgap. The parameter $a$ is the thickness of the $\mathrm{Si} / \mathrm{SiO}_{2}$ cladding pair.

\section{ACKNOWLEDGMENTS}

This research was sponsored by the Office of Naval Research (Y. S. Park), whose support is gratefully acknowledged.

\section{REFERENCES}

1. E. M. Purcell, "Spontaneous emission probabilities at radio frequencies," Phys. Rev. 69, p. 681, 1946.

2. Y. Yamamoto, S. Machida, and G. Björk, "Microcavity semiconductor laser with enhanced spontaneous emission," Phys. Rev. A 44, p. 657, 1991.

3. J. M. Gérard, B. Sermage, B. Gayral, B. Legrand, E. Costard, and V. Thierry-Mieg, "Enhanced spontaneous emission by quantum boxes in a monolithic optical microcavity," Phys. Rev. Lett. 81, p. 1110, 1998.

4. P. R. Berman, ed., Cavity Quantum Electrodynamics, Academic Press, San Diego, 1994.

5. V. Sandoghdar, F. Treussart, J. Hare, V. Lefévre-Seguin, J. M. Raimond, and S. Haroche, "Very low threshold whispering-gallery-mode microsphere laser," Phys. Rev. A 54, p. R1777, 1996.

6. A. Kiraz, P. Michler, C. Becher, B. Garyral, A. Imamoğlu, L. Zhang, E. Hu, W. V. Schoenfeld, and P. M. Petroff, "Cavity-quantum electrodynamics using a single inas quantum dot in a microdisk structure," Appl. Phys. Lett. 78, p. 3932, 2001.

7. O. Painter, R. K. Lee, A. Scherer, A. Yariv, J. D. O'Brien, P. D. Dapkus, and I. Kim, "Two-dimensional photonic band-gap defect mode laser," Science 284, p. 1819, 1999.

8. D. Brady, G. Papen, and J. E. Sipe, "Spherical distributed dielectric resonators," J. Opt. Soc. Am. B 10, p. 644, 1993.

9. M. A. Kaliteevski, S. Brand, R. A. Abram, and V. V. Nikolaev, "Optical eigenmodes of a multilayered spherical microcavity," J. Mod. Opt. 48, p. 1503, 2001. 
10. D. D. Smith and K. A. Fuller, "Photonic bandgaps in mie scattering by concentrically stratified spheres," J. Opt. Soc. Am. B 19, p. 2449, 2002.

11. P. Yeh, A. Yariv, and E. Marom, "Theory of a bragg fiber," J. Opt. Soc. Amer. 68, p. 1196, 1978.

12. Y. Xu, R. K. Lee, and A. Yariv, "Asymptotic analysis of bragg fibers," Opt. Lett. 25, p. 1756, 2000.

13. S. D. Hart, G. R. Maskaly, B. Temelkuran, P. H. Prideaux, J. D. Joannopoulos, and Y. Fink, "External reflection from omnidirectional dielectric mirror fibers," Science 296, p. 510, 2002.

14. J. G. Fleming, S.-Y. Lin, and R. Handley in Proceeding of the Solid-State Sensor Actuator and Microsystems Workshop, p. 173, (Hilton Head, S.C.), 2002.

15. J. D. Jackson, Classical Electrodynamics, John Wiley \& Sons, New York, third ed., 1999.

16. P. Yeh and A. Yariv, "Bragg reflection waveguides," Opt. Commun. 19, p. 427, 1976.

17. Y. Fink, J. N. Winn, S. Fan, C. Chen, J. Michel, J. D. Joannopoulos, and E. L. Thomas, "A dielectric omnidirectional reflector," Science 282, p. 1679, 1998.

18. M. P. van Exter, G. Niehuis, and J. P. Woerdman, "Two simple expression for the spontaneous emission factor," Phys. Rev. A 54, p. 3553, 1996.

19. A. Blanco, E. Chomski, S. Grabtchak, M. Ibisate, S. John, S. W. Leonard, C. Lopez, F. Meseguer, H. Miguez, J. P. Mondia, G. A. Ozin, O. Toader, and H. M. van Driel, "Large scale synthesis of a silicon photonic crystal with a complete three-dimensional bandgap near 1.5 micrometres," Nature 405, p. 437, 2000. 